\title{
Flavone-rich maize: an opportunity to improve the nutritional value of an important commodity crop
}

\author{
María I. Casas ${ }^{1,2,3}$, Silvia Duarte ${ }^{3,4}$, Andrea I. Doseff ${ }^{3,4}$ and Erich Grotewold ${ }^{1,3 *}$ \\ ${ }^{1}$ Center for Applied Plant Sciences, The Ohio State University, Columbus, OH, USA \\ 2 Molecular Cellular and Developmental Biology Graduate Program, The Ohio State University, Columbus, OH, USA \\ ${ }^{3}$ Department of Molecular Genetics, The Ohio State University, Columbus, OH, USA \\ ${ }^{4}$ Division of Pulmonary, Allergy, Critical Care, and Sleep, Department of Internal Medicine, Heart and Lung Research Institute, The Ohio State University, \\ Columbus, $\mathrm{OH}$, USA
}

\section{Edited by:}

Francesco Paolocci, Italian National Research Council (CNR) Institute of Plant Genetics Division of Perugia, Italy

\section{Reviewed by:}

Sixue Chen, University of Florida, USA

Milvia Luisa Racchi, University of Florence, Italy

\section{*Correspondence:}

Andrea I. Doseff, Heart and Lung Research Institute, The Ohio State University, 305B, 473 W 12th Avenue, Columbus, OH 43210, USA e-mail: doseff.1@osu.edu; Erich Grotewold, Department of Molecular Genetics, Center for Applied Plant Sciences, The Ohio State University, 012 Rightmire Hall, 1060 Carmack Rd., Columbus, $\mathrm{OH}$ 43210, USA

e-mail: grotewold.1@osu.edu
Agricultural outputs have resulted in food production continuously expanding. To satisfy the needs of a fast growing human population, higher yields, more efficient food processing, and food esthetic value, new crop varieties with higher caloric intake have and continue to be developed, but which lack many phytochemicals important for plant protection and adequate human nutrition. The increasing incidence of chronic diseases such as obesity, diabetes and cardiovascular diseases, combined with social disparity worldwide prompted the interest in developing enhanced crops that can simultaneously address the two sides of the current malnutrition sword, increasing yield while providing added nutritional value. Flavones, phytochemicals associated with the beneficial effects of the Mediterranean diet, have potent anti-inflammatory and anti-carcinogenic activities. However, many Mediterranean diet-associated vegetables are inaccessible, or lowly consumed, in many parts of the world. Maize is the most widely grown cereal crop, yet most lines used for hybrid maize production lack flavones. As a first step toward a sustainable strategy to increasing the nutritional value of maize-based diets, we investigated the accumulation and chemical properties of flavones in maize seeds of defined genotypes. We show that the pericarps of the P1-rr genotype accumulate flavones at levels comparable to those present in some flavone-rich vegetables, and are mostly present in their $C$ - and $O$-glycosylated forms. Some of these glycosides can be readily converted into the corresponding more active health beneficial aglycones during food processing. Our results provide evidence that nutritionally beneficial flavones could be re-introduced into elite lines to increase the dietary benefits of maize.

Keywords: maize, metabolism, nutrition, inflammation, cancer, nutritional disparity

\section{INTRODUCTION}

Malnutrition is widely spread worldwide, presented in diverse forms from undernourishment in people without access to minimum diet requirements, to people with access to processed foods rich in fats and sugars, dramatically increasing diet-related chronic diseases such as obesity, diabetes and cardiovascular diseases (Tanumihardjo et al., 2007; Martin et al., 2013). Hence, there is a growing interest to improve food nutritional value in order to fulfill minimum requirements, as well as to prevent diet-associated diseases.

The Mediterranean's diet consists of a largely plant-based dietary pattern associated with the low incidence of coronary heart disease, certain cancers and other diet-related illnesses, including obesity and diabetes (Stafford, 1990; Gates et al., 2009). Its composition includes legumes, nuts and olive oil,

Abbreviations: A7OG, apigenin-7-O-glucoside; L7OG, luteolin-7-O-glucoside; IV, isovitexin; IO, isoorientin; RIO, rhamnosylisoorientin. HPLC, high performance liquid chromatography; LC-MS/MS, liquid chromatography followed by tandem mass spectrometry; AME, acid whole maize extract; EME, enzymatically treated whole maize extract. making it markedly different from the Western-diet rich in saturated fatty acids derived from animal food (Trichopoulou and Vasilopoulou, 2000; Wahrburg et al., 2002). However, many components of the Mediterranean's diet are inaccessible or have a high cost, making other plants and plant-derived foods containing similar nutritional components potential targets to bring about improved nutritional value (Bonaccio et al., 2011).

Maize is one of the most important cereal crops worldwide, with the USA being the top producer. In order to satisfy the demands of a rapidly increasing population, most of the maize grown for feedstock, bio-ethanol and other industrial purposes are high-yield varieties grown at high densities. Additionally, to increase production, more efficient food processing methods have been developed (Morris and Sands, 2006). In order to fulfill these requirements, breeding has generated maize varieties that have looser husks, bigger endosperms, thinner pericarps and which have lost many of the phytochemicals important for plant protection, human and livestock nutrition (Bailey and Bailey, 1938; Huang et al., 2002). 
Amongst the phytochemicals lost during maize domestication and breeding are the flavonoids, plant specialized metabolites that play many important roles in plant growth and development, including pollen development and viability, UV protection, and insect pest deterrence (Grotewold, 2006; Falcone Ferreyra et al., 2012). Flavones, a flavonoid sub-family, have been associated with the beneficial effects of the Mediterranean diet. Specifically, the flavone apigenin, abundantly present in celery, parsley and other vegetables of the Mediterranean's diet (Justesen et al., 1998; Trichopoulou and Vasilopoulou, 2000), exhibits potent anti-angiogenic, anti-inflammatory and anti-carcinogenic activities (Vargo et al., 2006; Gonzalez-Mejia et al., 2010; Shukla and Gupta, 2010). Moreover, apigenin restores normal metabolic function during inflammatory conditions, through the regulation of mitochondrial function (Duarte et al., 2013). The identification of human cellular targets of apigenin provided new insights into the molecular mechanisms by which this flavone exerts its beneficial effects. Apigenin binds to the heterogeneous nuclear ribonucleoprotein A2 (hnRNPA2) and affects its multimerization, thereby inducing changes in splicing activity (Arango et al., 2013). Flavonoids have also been identified as potentially beneficial for feedstock nutrition, for example by reducing ruminal methanogenesis, by altering meat and milk fatty acid profiles, and by reducing lipid peroxidation in meats (Patra and Saxena, 2010; Vasta and Luciano, 2011).

Flavonoid biosynthesis begins with the condensation of three malonyl-CoA molecules with $p$-coumaroyl-CoA by chalcone synthase [abbreviated CHS, encoded by the colorless2 (C2) locus], to form naringenin chalcone. Subsequently, chalcone isomerase (CHI) converts narigenin chalcone into the flavanone naringenin, generating a key branching step in the pathway (Winkel-Shirley, 2001; Grotewold, 2006). Naringenin can serve as substrate for the flavanone- $3^{\prime}$-hydroxylase enzyme, encoded by the locus $\operatorname{Pr} 1$, to generate eriodictyol. Naringenin is then converted into various flavones by a combination of steps involving a flavanone2-hydroxylase $(\mathrm{F} 2 \mathrm{H})$ and a $C$-glycosyl transferase (CGT), to generate apigenin-6- $C$-glucoside (or its isomer, apigenin-8- $C$ glucoside), also known as isovitexin and vitexin, respectively. In the case of eriodictyol, the flavones generated after these conversions steps are luteolin-6-C-glucoside (or its isomer, luteolin-8-C-glucoside), namely isoorientin and orientin, respectively (Winkel-Shirley, 2001; McMullen et al., 2004; Morohashi et al., 2012; Falcone Ferreyra et al., 2013). Flavones can also be generated from naringenin by means of a 2-oxoglutarate dependent dioxygenase (2-ODD), as is the case for Apiaceae like celery (Yan et al., 2014). Flavones are often $O$ - or $C$ - glycosylated by glycosyl transferases, to generate, for instance, apigenin-7-Oglucoside (A7OG, Figure 1).

The R2R3-MYB transcription factor PERICARP COLOR 1 (P1) controls the accumulation of the brick red phlobaphene pigments in the pericarp (Styles and Ceska, 1977; Grotewold et al., 1991b) the outermost layer of the kernel, a maternal tissue that corresponds to the modified ovary wall (Kiesselbach, 1949). Phlobaphenes are polymers of the flavan-4-ols apiforol or luteoforol, generated from naringenin or eriodictyol by dihydroflavonol reductase (DFR), encoded by maize $A 1$. Different $P 1$ alleles specify different pericarp and cob glume colors. For example, $P 1-w w$ results in white pericarps and white cob glumes, whereas $P 1-r r$ specifies red pericarps and red cob glumes (Figure 2). P1-wr and P1-rw alleles responsible for white pericarps and red cobs, or red pericarps and white cobs respectively, have also been described (Anderson, 1924; Chopra et al., 1996). A1 mutants in a P1-rr background (P1-rr;a1) display an unidentified brown pigment that contrasts with the white $P 1-w w$ pericarps, suggesting metabolic shunting toward a different branch of the flavonoid pathway (Styles and Ceska, 1977). Additionally, $P 1$ has been identified as a major QTL for $C$-glycosylflavone accumulation in maize silks; in the absence of a functional $A 1$ allele, $P 1-r r$ pericarps accumulate C-glycosylflavones (Styles and Ceska, 1977; Byrne et al., 1996). Most commercial high-yield hybrid maize lines are colorless in the pericarp and lack flavones. As is the case for sweet corn and other commercial varieties, maize kernels contain over $25 \%$ sugar (Singh et al., 2014), resulting in maize being partially responsible for the poor nutritional value combined with high caloric intake of many processed foods. For example, high fructose corn syrup has been associated with high incidence of metabolic syndrome, diabetes, kidney damage and liver disease (Ferder et al., 2010).

As a first step toward a rational strategy to increase the nutritional value of maize-based diets, we investigated the accumulation and chemical properties of flavones in defined maize genotypes. We show here that the pericarps of P1-rr maize accumulate flavones at levels comparable to those present in flavone-rich vegetables. We found that a significant portion of these flavones is present as $C$ - and $O$-glycosylated derivatives. We describe strategies that permit the conversion of some of these glycosides into the more bioactive aglycones in a whole food context. The levels of flavones in various maize lines, including P1-rr;a1, were also quantified. Together, our results provide evidence that nutritionally beneficial flavones could be reintroduced into maize elite lines to increase the dietary benefits of maize products.

\section{MATERIALS AND METHODS MAIZE STOCKS AND PLANT MATERIALS}

The P1-rr 65-CFS-305 and P1-ww 4Co43 lines were introgressed into A619 $(P 1-w w)$ as previously described (Morohashi et al., 2012). The P1-rr;al stock has the $P 1^{4 B-2}$ allele into which the $a 1:: r d-t$ sh 2 mutant allele was introduced by crossing (Grotewold et al., 1991a; Pooma et al., 2002). It is not known however, whether the $A 1$ allele in the P1-rr 65-CFS-305 line is the same as in P1-rr;a1. It was also noted that the kernel and ear sizes from P1-rr;a1 were smaller, compared to those of the other lines in this study. In all cases, pericarps and whole kernels were collected at different developmental times including 14, 25, and 40 days after pollination (DAP) and saved at $-80^{\circ} \mathrm{C}$ for further analyses. Sweet corn ears were obtained from various commercial providers at milky stage (approximately 18 DAP).

\section{CHEMICALS}

Apigenin, A7OG, luteolin, L7OG, vitexin (apigenin-8-Cglucoside), isovitexin (apigenin-6- $C$-glucoside), luteolin and orientin (luteolin-8-C-glucoside) standards were obtained from Sigma (Sigma Aldrich, St. Louis, MO); isoorientin (luteolin6-C-glucoside) was kindly provided by Dr. Michael McMullen 


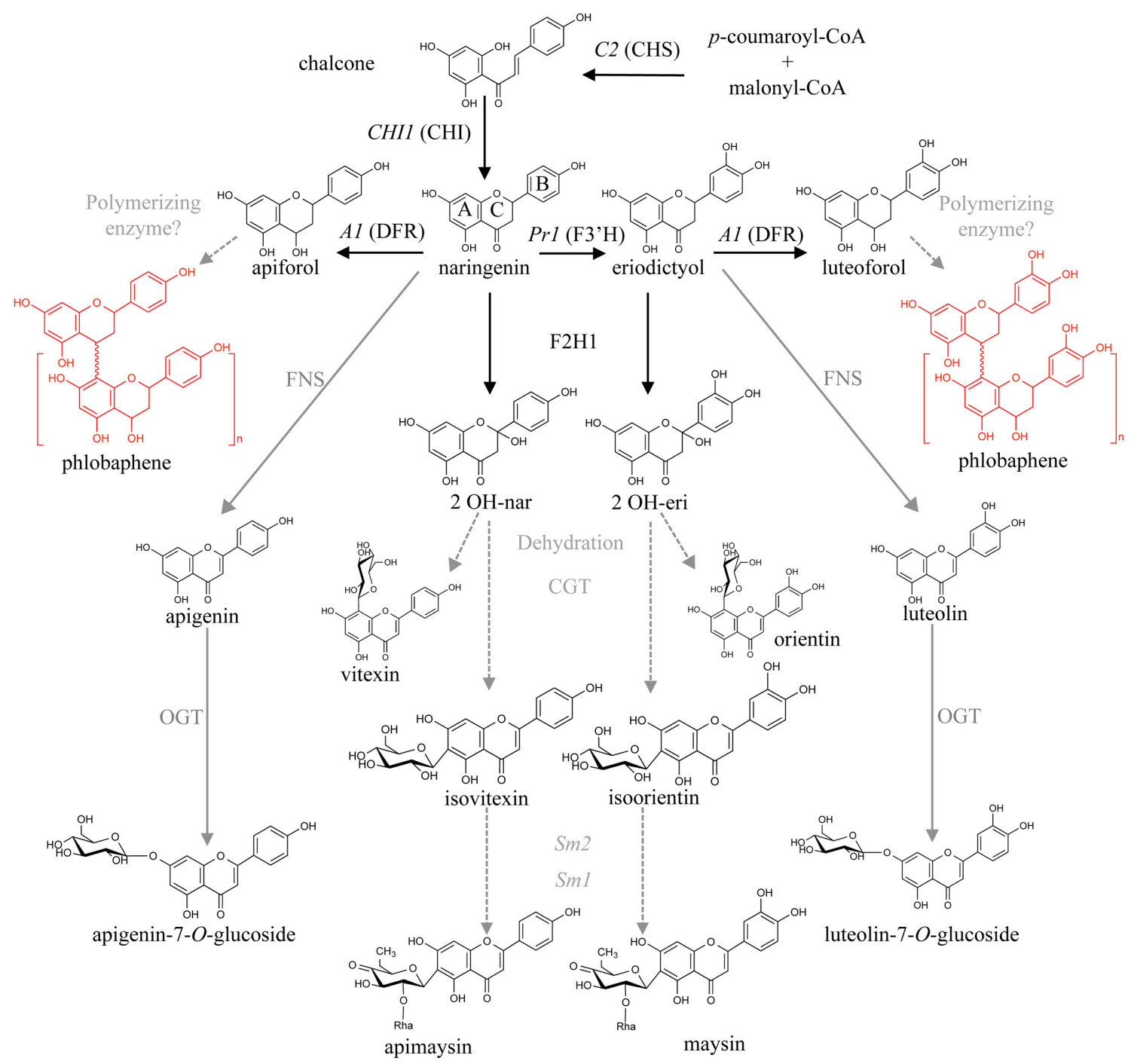

FIGURE 1 | Proposed maize flavone and 3-deoxy flavonoid biosynthetic pathways. Condensation of $p$-coumaroyl-CoA and malonyl-CoA by chalcone synthase (referred to as $\mathrm{CHS}$, encoded by the locus $\mathrm{C} 2$ ) results in naringenin chalcone, which is then converted to naringenin by chalcone isomerase ( $\mathrm{CHI}$ ). Naringenin is converted to apiforol (flavan-4-ol) by a dihydroflavonol reductase (DFR, A1) and subsequently polymerized into phlobaphenes. It can also be converted to isovitexin ( $C$-glycosylflavone) by a flavanone-2-hydrohylase (F2H) and a C-glycosyl transferase (CGT). A flavone synthase (FNS) could also catalyze this step followed by an O-glycosyl transferase (OGT). Naringenin can also be converted to eriodictyol by a flavanone-3'-hydroxylase $\left(\mathrm{F}^{\prime}{ }^{\prime} \mathrm{H}, \operatorname{Pr} 1\right)$. The proposed steps for conversion of apigenin and luteolin into the $C$-glycosylflavones apimaysin and maysin respectively, involve at least three enzymatic conversions: glycosylation at C-6, followed by a rhamnosylation and dehydration steps mediated by the Sm2 and Sm1 loci (adapted from Morohashi et al., 2012). Enzymes identified in black, those proposed in gray.
(USDA-ARS, Columbia, MI). Methanol and acetonitrile were obtained from Sigma (Sigma Aldrich) and ethanol was obtained from Decon Labs, Inc. (King of Prussia, PA). $\mathrm{HCl}, \mathrm{H}_{3} \mathrm{PO}_{4}, \mathrm{KOH}$ were obtained from Fisher Scientific (Fair Lawn, NJ).

\section{FLAVONOID EXTRACTION FROM MAIZE PERICARPS}

Fresh pericarps were dissected at 14, 25, and 40 DAP and freeze-dried overnight. Dried pericarps were extracted with $100 \%$ methanol over-night at $-20^{\circ} \mathrm{C}$ using $50 \mathrm{mg}$ of dry pericarps per $\mathrm{ml}$ of methanol. Following incubation, half the volume of the methanolic extracts were used for further analyses (nonhydrolyzed), and the other half was dried under vacuum and re-suspended in $5 \%(\mathrm{v} / \mathrm{v}) \mathrm{HCl}$ in butanol and boiled for $1 \mathrm{~h}$ (hydrolyzed) prior to analyses.

\section{MAIZE WHOLE-FOOD EXTRACT PREPARATION}

Maize extracts were prepared as similarly described (Hostetler et al., 2012). Briefly, kernels stored at $-80^{\circ} \mathrm{C}$ were macerated with mortar and pestle and incubated for $3 \mathrm{~h}$ at room temperature, subsequently frozen in liquid $\mathrm{N}_{2}$ and lyophilized overnight. Kernels were incubated for $3 \mathrm{~h}$ following the same protocol as previously used for celery (Hostetler et al., 2012), which was used as a positive control for extract preparation. The lyophilized material was ground, re-suspended in $1.5 \mathrm{~N} \mathrm{H}_{3} \mathrm{PO}_{4}$ (at 1 gr per $17 \mathrm{ml}$ ) 


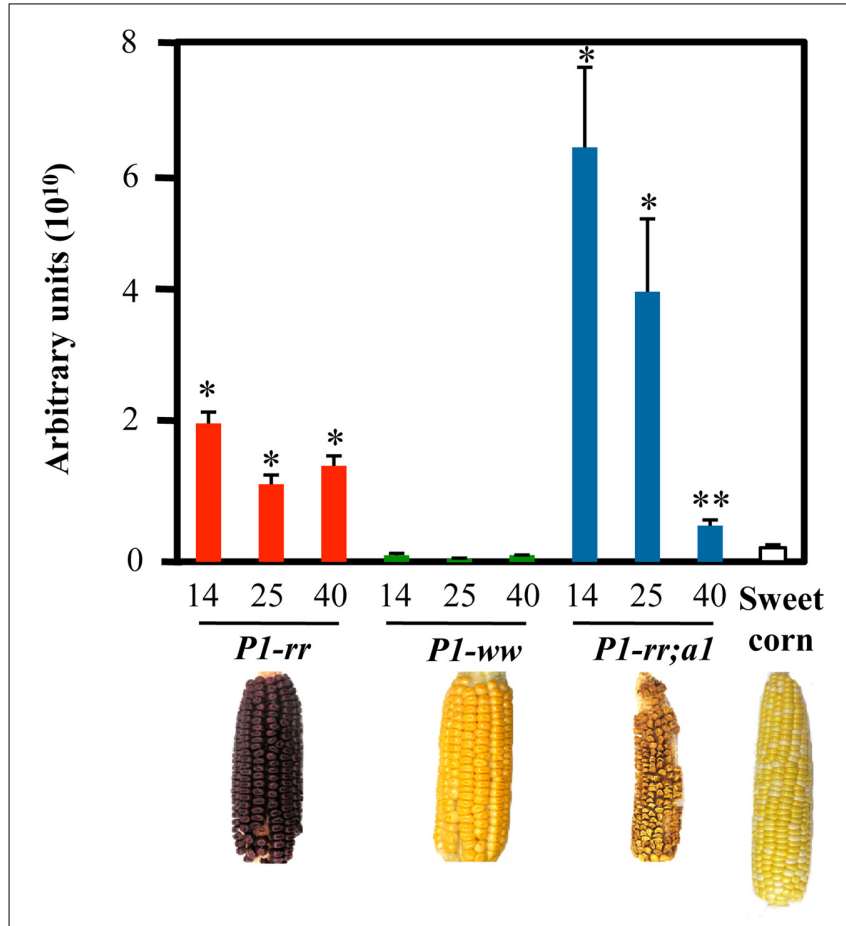

FIGURE 2 | Higher flavonoid levels in $\boldsymbol{P} 1$ expressing lines. HPLC analyses of pericarps at 14,25 , and 40 DAP. Total flavonoid levels are indicated as arbitrary units (mean values \pm s.e.m., $n=5$ for all maize lines and $n=3$ for $P 1$-rr;a 1). $t$-test $* p<0.01$ or ${ }^{* *} p<0.05$ compared to sweet corn. Arbitrary units correspond to total peak area at $350 \mathrm{~nm}$ per $\mathrm{g}$ of dry pericarp.

and boiled for $90 \mathrm{~min}$, before neutralizing with $10 \% \mathrm{KOH}$. This extract is referred hereafter as AME (acid whole maize extract). In addition, the mix was incubated with commercially available ground raw almond (Whole Foods Market, Columbus, $\mathrm{OH} ; 17 \%$ dry weight of almond over $100 \mathrm{~g}$ of the lyophilized material) for $2 \mathrm{~h}$ at $50^{\circ} \mathrm{C}$ to convert the $\mathrm{O}$-glucosides to aglycones. The resulting mixture was then lyophilized, and $1 \mathrm{gr}$ was extracted twice with $5 \mathrm{ml}$ ethanol $70 \%(\mathrm{v} / \mathrm{v})$. This extract was referred hereafter as EME (enzymatically treated whole maize extract). An aliquot of both AME and EME extracts were dried under $\mathrm{N}_{2}$ gas and reconstituted in 100\% methanol for LC-MS/MS analyses.

\section{HPLC ANALYSES}

Pericarps and maize whole-food methanolic extracts were filtered through a $0.2 \mu \mathrm{m}$ membrane (Pall Nanosep ${ }^{\circledR} \mathrm{MF}$ ) prior injecting $20 \mu \mathrm{l}$ into a W2690-5 Waters (Milford, MA) separation module and a W2996 photodiode array (PDA) module following absorbance at $350 \mathrm{~nm}$, corresponding to the maximum absorbance wavelength for flavones (Snook et al., 1989) and flavonols (Anouar et al., 2012), using a Symmetry C18 reversephase column ( $3.5 \mu \mathrm{m}, 4.9 \mathrm{~mm}, 75 \mathrm{~mm}$, Waters). Running conditions were conducted as previously described (Casati and Walbot, 2005), briefly, an initial gradient of $80 \%$ solvent A-20\% solvent B (solvent A: water; solvent B: acetonitrile) with a $0.75 \mathrm{ml} / \mathrm{min}$ flow to $100 \%$ solvent $B$ at $15: 33 \mathrm{~min}$ and kept for 1:20 min to finally return to initial conditions at 18:00 min. Total flavonoid levels were expressed as total peak area at $350 \mathrm{~nm}$ per g dry weight of pericarp tissue, referred to as arbitrary units in Figure 2. A fresh kernel weights $\sim 150 \mathrm{mg}$ at 14 DAP and the pericarp corresponds to $\sim 20 \%$ of the total fresh weight at this developmental stage. All statistical analyses were done using two-tailed Student's $t$-test.

\section{FLAVONE LC-MS/MS ANALYSES}

Hydrolyzed and non-hydrolyzed pericarp methanolic extracts and maize whole-food methanolic extracts were used for flavone analyses performed by liquid chromatography followed by tandem mass spectrometry (LC-MS/MS) on a Linear Ion Trap Quadrupole LC-MS/MS with a Q-Trap 5500 Mass Spectrometer (AB-SciEX) at the Center for Applied Plant Sciences (CAPS) Targeted Metabolomics Laboratory (TML, http://metabolomics. osu.edu/). LC was performed on a Symmetry C18 column $(3.5 \mu \mathrm{m}, 4.9 \mathrm{~mm}, 75 \mathrm{~mm}$, Waters) injecting $15 \mu \mathrm{l}$ with a flow of $1 \mathrm{ml} / \mathrm{min}$, solvent A: water, solvent B: acetonitrile following previously established protocols (Falcone Ferreyra et al., 2013). Briefly, an initial gradient of $20 \%$ solvent $\mathrm{A}-80 \%$ solvent $\mathrm{B}$ (solvent A: water; solvent $\mathrm{B}$ : acetonitrile) from 0.00 to $2.00 \mathrm{~min}$; followed by $7.00 \mathrm{~min}$ of 20 to $60 \% \mathrm{~A}$; then 9.10 to $11.00 \mathrm{~min}$ to $90 \% \mathrm{~A} ; 0.1 \mathrm{~min}$ decrease from 90 to $20 \%$ A and finally 11.10 to 14.00 min $20 \%$ A was used. MS spectra were acquired using electrospray ionization (ESI) and Multiple Reaction Monitoring on the negative mode. Conditions and retention times for each compound are summarized in Supplementary Table 1. Flavone quantifications were performed based on the corresponding standard curves (Supplementary Figure 1). Limits of detection, limits of quantification, correlation coefficients $\left(\mathrm{R}^{2}\right)$, and formula weights (in atomic mass units, amu) for each flavone are summarized in Supplementary Table 2.

\section{RESULTS}

\section{KERNELS OF P1 EXPRESSING MAIZE LINES HAVE HIGH FLAVONOID CONTENT}

Flavonoid content was evaluated in pericarps of different maize lines including P1-rr, P1-ww, P1-rr;al and commercial sweet corn, at three different developmental stages: 14 days after pollination (DAP), a developmental point close to the milky stage, when corn is normally harvested for human consumption, 25 and 40 DAP, the latter time corresponding to a stage used for longterm seed storage or livestock feed. We found that the majority of the flavonoids analyzed had very short retention times ranging from 1.8 to $4.8 \mathrm{~min}$ by HPLC (Supplementary Figure 2) and most likely correspond to glycosides (Bligh et al., 2013). P1-rr;a1 showed the highest amount of total flavonoids, as compared with P1-rr, P1-ww and sweet corn (Figure 2), in agreement with previous studies (Styles and Ceska, 1989). The levels of flavonoids in P1-rr;a1 were 3 fold higher than P1-rr at 14 DAP $\left(6.4 \times 10^{10}\right.$ AU compared to $2.13 \times 10^{10}$ respectively), P1-rr;al levels were 64 fold higher than $P 1-w w\left(1.0 \times 10^{9} \mathrm{AU}\right)$ and 29 fold higher than sweet corn $\left(2.2 \times 10^{9} \mathrm{AU}\right)$ at the same developmental stage. At 25 DAP, P1-rr;al levels were 3 fold higher than P1-rr $\left(4.2 \times 10^{10}\right.$ vs. $\left.1.2 \times 10^{10} \mathrm{AU}\right)$ and 92 fold higher than $P 1-w w\left(4.5 \times 10^{8}\right.$ $\mathrm{AU})$. Finally, at $40 \mathrm{DAP}, \mathrm{P1-rr;a1}$ levels dropped to $5.6 \times 10^{9}$ $\mathrm{AU}$ whereas the levels of P1-rr were $1.5 \times 10^{10} \mathrm{AU}$ and $P 1-w w$ 
$1.0 \times 10^{9} \mathrm{AU}$, respectively (Figure 2). These results show that the P1-rr;a1 genotype has a significantly higher flavonoid content at early harvest times, providing a window of opportunity for the development of flavonoid-rich maize whole foods.

\section{FLAVONES ARE PRESENT PRIMARILY AS $\boldsymbol{C}$ - AND 0 -GLUCOSIDES IN MAIZE KERNELS}

To identify the contribution of flavones to the absorbance observed at $350 \mathrm{~nm}$ in the various maize genotypes, liquid chromatography followed by tandem mass spectrometry (LC-MS/MS) was performed on pericarp extracts of the various maize lines at 14 and 25 DAP and in sweet corn at milky stage (shown in Figure 3 for apigenin). P1-ww and sweet corn kernels showed the lowest levels of total flavones, ranging between $\sim 0.6-1.3 \mu \mathrm{g}$ flavones per $100 \mathrm{gr}$ fresh kernel respectively (Table 1 ), consistent with the results obtained by HPLC (Figure 2). P1-rr accumulated 360 and $71 \mu \mathrm{g}$ of total flavone per $100 \mathrm{gr}$ of fresh kernel at 14 and 25 DAP, respectively. Notably, the introduction of the a1 allele into $P 1-r r$ significantly increased $(\sim 100$ fold $)$ the levels of total flavone, resulting in 36 and $25 \mathrm{mg}$ of total flavone per $100 \mathrm{gr}$ of fresh kernel at 14 and 25 DAP, respectively (Table $\mathbf{1}$ ). We found that in all the lines, flavone content decreased during development. This is likely a consequence of the diversion of naringenin toward flavan-4-ol formation, which will polymerize into phlobaphenes in P1-rr, or into the brown pigment in P1-rr;al, as pericarp development continues.

P1-rr;a1 had the highest contents of apigenin and luteolin at both developmental stages $(2$ and $18 \mu \mathrm{g} / 100 \mathrm{gr}$ fresh weight at 14 $\mathrm{DAP}$, and 0.54 and $8 \mu \mathrm{g} / 100 \mathrm{gr}$ fresh weight at $25 \mathrm{DAP}$, respectively), when compared to the other maize lines. Unexpectedly, we found that apigenin and luteolin were similar in P1-rr and P1-ww or sweet corn at both developmental stages (Table 1). The difference between apigenin and luteolin levels at both developmental stages in P1-rr;a1 could be explained by the presence of the $\operatorname{Pr} 1$ locus, encoding a flavanone- $3^{\prime}$ hydroxylase (Sharma et al., 2011), responsible for adding a hydroxy group to $\mathrm{C} 3$ on the $\mathrm{B}$-ring of the flavonoid backbone (Figure 1).

Apigenin-7-O-glucoside (A7OG) and luteolin-7-O-glucoside (L7OG) contents were similarly low in P1-ww and sweet corn (Table 1). A7OG levels at 14 DAP were 5 fold higher in $\mathrm{P1-rr}$ as compared with P1-rr;al, accumulating $\sim 13$ and $2 \mu \mathrm{g} / 100 \mathrm{gr}$ fresh weight, respectively. At $25 \mathrm{DAP}$, this difference is smaller, with P1-rr;a1 accumulating more A7OG than P1-rr. L7OG was remarkably ( $\sim 340$ fold) higher in P1-rr;al compared to $P 1-r r$ at 14 DAP (1023 vs. $3 \mu \mathrm{g} / 100 \mathrm{gr}$ fresh weight), and $\sim 450$ fold higher at 25 DAP (Table 1).

The apigenin $C$-glucosides vitexin and isovitexin were barely detectable in sweet corn and P1-ww at both developmental stages (Table 1). They accumulate at similar levels in P1-rr and P1-rr;a1 at 14 DAP (8 and $9 \mu \mathrm{g} / 100 \mathrm{gr}$ fresh weight, and 9 and $7 \mu \mathrm{g} / 100 \mathrm{gr}$ fresh weight, respectively) and no significant changes were observed in these compounds at 25 DAP in P1rr;al, whereas approximately a 6 fold decrease is observed for P1-rr (Table 1). The luteolin-derived $C$-glucosides, orientin and isoorientin, are found more abundantly in P1-rr;a1 at both developmental stages, with isoorientin reaching almost an order of magnitude higher than orientin $(\sim 1500 \mu \mathrm{g}$ vs. $\sim 120 \mu \mathrm{g} / 100 \mathrm{gr}$

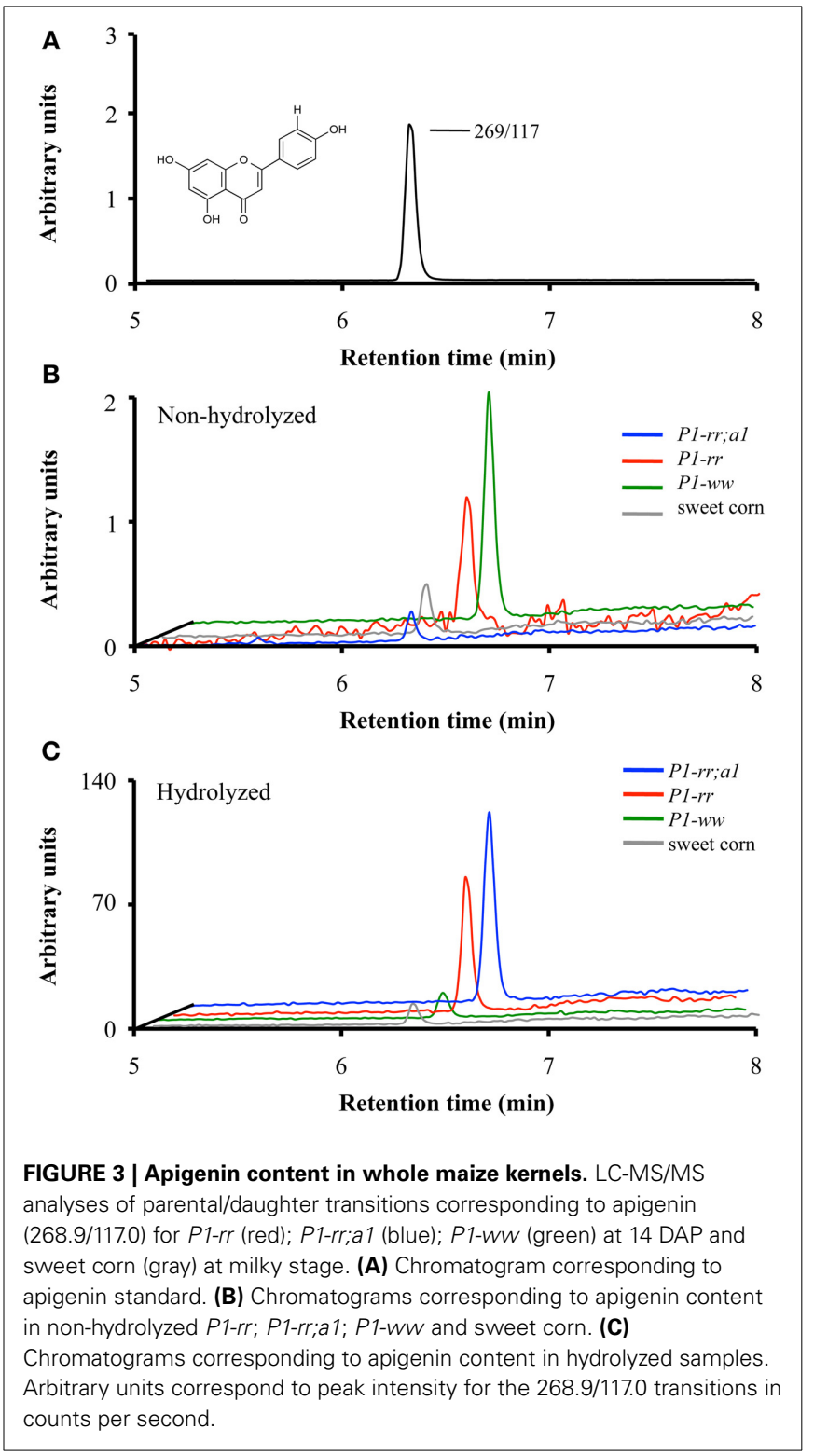

fresh weight, respectively). In both cases, no differences are observed at 14 and 25 DAP. Orientin and isoorientin are present in $\mathrm{P1}-\mathrm{rr}$ at reasonably high levels, but correspond to 1 or $10 \%$ respectively to the levels present in P1-rr;a1 (Table 1).

Rhamnosylisoorientin (RIO), the precursor for the strong insect deterrent compound maysin (Byrne et al., 1998), contains the luteolin backbone (Figure 1). Our results indicate that $P 1-w w$ and sweet corn have low but similar levels of RIO and maysin $(\sim 0.01-0.09 \mu \mathrm{g} / 100 \mathrm{gr}$ fresh weight). P1-rr has significantly higher levels of RIO and maysin, reaching $\sim 62$ and $246 \mu \mathrm{g} / 100 \mathrm{gr}$ fresh weight at $14 \mathrm{DAP}$, and these levels decrease by more than $50 \%$ by 25 DAP ( 16 and $46 \mu \mathrm{g} / 100 \mathrm{gr}$ fresh weight, respectively). In P1-rr;a1, a remarkably higher content of both RIO and maysin is observed, reaching $\sim 7$ and $8 \mathrm{mg} / 100 \mathrm{gr}$ fresh weight for RIO at both developmental stages and as high as $\sim 26 \mathrm{mg} / 100 \mathrm{gr}$ fresh weight at 14 DAP for 
Table 1 | Flavone content in non-hydrolyzed whole maize kernel.

\begin{tabular}{|c|c|c|c|c|c|c|c|}
\hline & \multicolumn{2}{|c|}{$P 1-w w^{a}$} & \multicolumn{2}{|c|}{$P 1-r r^{b}$} & \multicolumn{2}{|c|}{ P1-rr;a1 } & Sweet corn ${ }^{d}$ milky stage \\
\hline Luteolin & $0.03 \pm 0.01$ & $0.010 \pm 0.003$ & $0.20 \pm 0.06$ & $0.07 \pm 0.02$ & $18 \pm 5$ & $8 \pm 4$ & $0.05 \pm 0.03$ \\
\hline A7OG & $0.02 \pm 0.01$ & $<0.005$ & $13 \pm 5$ & $1.0 \pm 0.3$ & $2.4 \pm 1.1$ & $5 \pm 2$ & $0.030 \pm 0.002$ \\
\hline L7OG & $0.03 \pm 0.02$ & $0.020 \pm 0.003^{*}$ & $3.0 \pm 0.6^{*}$ & $0.7 \pm 0.1$ & $1023 \pm 342$ & $453 \pm 189$ & $0.06 \pm 0.01$ \\
\hline 10 & $0.05 \pm 0.02$ & $0.010 \pm 0.003$ & $10 \pm 3$ & $2.2 \pm 0.5$ & $1552 \pm 653$ & $1369 \pm 648$ & $0.6 \pm 0.5$ \\
\hline Orientin & $<0.005$ & $<0.005$ & $9 \pm 2$ & $1.6 \pm 0.3$ & $116 \pm 41$ & $85 \pm 32$ & $0.07 \pm 0.05$ \\
\hline $\mathrm{RIO}$ & $0.010 \pm 0.004^{*}$ & $0.010 \pm 0.003^{*}$ & $62 \pm 20$ & $16 \pm 6$ & $6779 \pm 3265$ & $7908 \pm 4408$ & $0.09 \pm 0.02$ \\
\hline Maysin & $0.02 \pm 0.01$ & $<0.005$ & $246 \pm 51^{*}$ & $46 \pm 4$ & $26,291 \pm 12,340$ & $14,694 \pm 7132$ & $0.23 \pm 0.16$ \\
\hline Total & $0.6 \pm 0.3$ & $0.08 \pm 0.02$ & $360 \pm 85$ & $71 \pm 12$ & $36,000 \pm 17,000$ & $25,000 \pm 12,000$ & $1.3 \pm 0.9$ \\
\hline
\end{tabular}

Flavone content was determined by LC-MS/MS using MRM in the negative mode following parental/daughter transitions corresponding to flavones in their aglycone and glycosylated forms. Results are indicated as $\mu \mathrm{g}$ of flavones/100 grams of fresh kernels. $t$-test values ${ }^{*} p<0.01$ compared to sweet corn. Mean values \pm s.e.m.; biological replicates for $a: n=6 ; b: n=5 ; c$ and $d: n=3$. A7OG, apigenin-7-O-glucoside; L7OG, Iuteolin-7-O-glucoside; IV, isovitexin; IO, isoorientin and RIO, rhamnosylisoorientin.

maysin, that decreased to almost half at 25 DAP (Table 1). Taken together, these findings show that the levels of flavones can be significantly improved by the presence of the P1 transcription factor in P1-rr maize lines, and that this effect is even more pronounced in lines that have the recessive al allele.

\section{ACID HYDROLYSIS INCREASES MAIZE KERNEL FLAVONE AGLYCONE CONTENT}

To determine whether acid hydrolysis would increase flavone aglycone content, LC-MS/MS analyses were performed on hydrolyzed lysates of pericarps corresponding to the different maize lines (apigenin shown in Figure 3). As we determined before hydrolysis (Table 1), P1-rr;al hydrolyzed lysates show the highest total flavone content at both developmental stages, when compared with all the other maize lines analyzed $(\sim 12 \mathrm{mg} / 100 \mathrm{gr}$ fresh weight, Table 2). P1-rr also has high levels, yet $\sim 10$ fold lower than in P1-rr;a1 (900 $\mu \mathrm{g} / 100 \mathrm{gr}$ fresh weight, Table 2). No significant differences in the total levels of hydrolyzed flavones were observed in P1-rr;al at 14 and 25 DAP, whereas a 3 fold decrease was observed for P1-rr at 25 DAP. In contrast, both P1$w w$ and sweet corn have similarly lower levels of total flavones, which remained stable during development (9 and $17 \mu \mathrm{g} / 100 \mathrm{gr}$ fresh weight, respectively) (Table 2 ).

The hydrolysis of P1-rr kernel extracts at 14 DAP shows a 110 fold increase in apigenin and a 65 fold increase in luteolin (36 and $13 \mu \mathrm{g} / 100 \mathrm{gr}$ fresh weight, respectively), and these levels of flavones are about half of those observed in 25 DAP kernel hydrolyzed lysates. In P1-rr;a1, the aglycone luteolin is found more abundantly, reaching $\sim 525$ and $275 \mu \mathrm{g} / 100 \mathrm{gr}$ fresh weight at 14 and 25 DAP, respectively, whereas apigenin levels are 30 times lower $(16 \mu \mathrm{g} / 100 \mathrm{gr}$ fresh weight at both developmental stages; Table 2). Hydrolysis of P1-ww and sweet corn extracts results in an increase of aglycones of around one order of magnitude (Table 2), likely derived from the low levels of glycosides present in these lines (Table 1).
In the case of A7OG, a different distribution is found in both P1-rr;a1 and P1-rr, as compared with P1-ww or sweet corn. A7OG content is reduced by $\sim 10$ fold in hydrolyzed extracts obtained from 14 DAP P1-rr and P1-rr a1 kernels. In contrast, increased levels are observed in both $P 1-w w$ and sweet corn $(0.2$ and $0.4 \mu \mathrm{g} / 100 \mathrm{gr}$ fresh weight at $14 \mathrm{DAP}$; blue boxes in Table 2). If the acid hydrolysis would have been complete, A7OG and L7OG should have disappeared from the hydrolyzed samples, or their levels should have been significantly reduced, which is not always the case (Table 2). It is possible that we misidentified A7OG and L7OG. However, we do not think that this is likely because we used pure commercial standards for these two compounds, which show over a minute difference in their retention times, and they present different parental/daughter ion combinations (Supplementary Table 1). Alternatively, it is possible that the hydrolysis was not complete, despite the strong conditions used. The increase in both flavone glucosides with hydrolysis can be explained by a more decorated flavone, perhaps not present among the ones studied here (Table 1), being partially hydrolyzed into A7OG or L7OG. This is consistent with the significant increase in isoorientin after hydrolysis; none of the flavones described in Table 1 was found to be high enough in sweet corn to provide the levels of this $C$-glycosylflavone (7 $\mu \mathrm{g} / 100 \mathrm{gr}$ fresh weight) found after hydrolysis (Table 2).

L7OG decreases by $\sim 7$ and 2.5 fold at 14 and 25 DAP, respectively in P1-rr;a1 (Tables 1, 2). However, in P1-ww and sweet corn, L7OG shows an increase of $\sim 10$ fold at each developmental stage $(0.5$ and $0.2 \mu \mathrm{g} / 100 \mathrm{gr}$ fresh weight at 14 and 25 DAP, respectively). In P1-rr, L7OG levels increase $\sim 3$ fold at 14 DAP and 6 fold at 25 DAP (Table 2, gray boxes), reaching 8 and $4 \mu \mathrm{g} / 100 \mathrm{gr}$ fresh weight, respectively. Hydrolysis increases the levels of the apigenin $C$-glucosides vitexin and isovitexin in all the samples, at both developmental stages (Table 2). P1-rr shows the highest levels at 14 DAP ( 89 and $84 \mu \mathrm{g} / 100 \mathrm{gr}$ fresh weight) corresponding to a 10 -fold increase (Table 2, gray boxes and underlined values), and the increase was even larger $(\sim 15$ fold $)$ 
Table 2 | Flavone content in hydrolyzed whole maize kernel.

\begin{tabular}{|c|c|c|c|c|c|c|c|}
\hline & \multicolumn{2}{|c|}{$P 1-w w^{a}$} & \multicolumn{2}{|c|}{$P 1-r r^{b}$} & \multicolumn{2}{|c|}{ P1-rr;a $1^{c}$} & Sweet corn ${ }^{d}$ milky stage \\
\hline Apigenin & $3 \pm 2$ & $2 \pm 1$ & $36 \pm 4^{*}$ & $11 \pm 2$ & $16 \pm 1$ & $16 \pm 1$ & $5 \pm 3$ \\
\hline Luteolin & $0.8 \pm 0.4$ & $0.4 \pm 0.2$ & $13 \pm 1^{*}$ & $7 \pm 2$ & $525 \pm 4$ & $275 \pm 8$ & $2.0 \pm 0.6$ \\
\hline A7OG & $0.2 \pm 0.1$ & $0.10 \pm 0.07$ & $1.0 \pm 0.3$ & $0.8 \pm 0.2$ & $0.50 \pm 0.02$ & $1.0 \pm 0.1$ & $0.4 \pm 0.2$ \\
\hline L7OG & $0.5 \pm 0.2$ & $0.2 \pm 0.1$ & $8 \pm 1^{*}$ & $4.0 \pm 0.5^{*}$ & $146 \pm 9^{*}$ & $179 \pm 15$ & $0.8 \pm 0.3$ \\
\hline 10 & $0.8 \pm 0.3$ & $0.4 \pm 0.2^{*}$ & $600 \pm 200$ & $240 \pm 74$ & $\underline{11,000 \pm 1500}$ & $12,000 \pm 2000^{*}$ & $7 \pm 2$ \\
\hline Orientin & $0.10 \pm 0.06$ & $0.03 \pm 0.01 *$ & $56 \pm 21$ & $13 \pm 7$ & $\underline{713 \pm 429}$ & $\underline{271 \pm 32^{*}}$ & $0.9 \pm 0.3$ \\
\hline $\mathrm{RIO}$ & $1.0 \pm 0.3$ & $0.4 \pm 0.1$ & $10 \pm 3$ & $5 \pm 2$ & $38 \pm 18$ & $37 \pm 9^{*}$ & $1.0 \pm 0.4$ \\
\hline Maysin & $2 \pm 1$ & $0.7 \pm 0.4$ & $1.5 \pm 0.5$ & $0.5 \pm 0.1$ & $24 \pm 7$ & $4.0 \pm 0.5^{*}$ & $0.70 \pm 0.07$ \\
\hline Total & $9 \pm 4$ & $4 \pm 2$ & $900 \pm 300$ & $324 \pm 95$ & $12,000 \pm 2000$ & $13,000 \pm 2000$ & $17 \pm 7$ \\
\hline
\end{tabular}

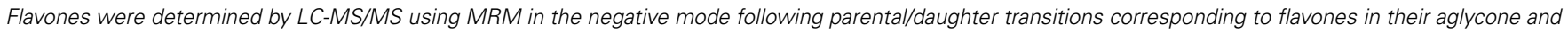

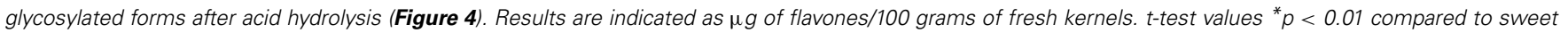

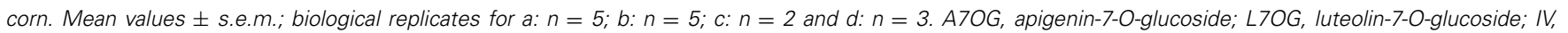
isovitexin; IO, isoorientin and RIO, rhamnosylisoorientin.

at 25 DAP (Table 2 gray boxes and underlined values), reaching $\sim 23 \mu \mathrm{g} / 100 \mathrm{gr}$ fresh weight for both compounds. Vitexin and isovitexin levels also increase during hydrolysis, but to a lower level in P1-rr;a1 at both 14 and 25 DAP (Table 2, gray boxes and underlined values), resulting in $2-4$ fold differences, when compared with the non-hydrolyzed material (Table 1). In P1-ww, at both developmental stages as well as in sweet corn, the levels increase, but remain always significantly lower than in P1-rr and P1-rr;a1. Orientin presented the highest levels in P1-rr;al at both developmental stages (underlined and in gray in Table 2), with $0.71 \mathrm{mg}$ per $100 \mathrm{gr}$ fresh weight at $14 \mathrm{DAP}$ and $0.27 \mathrm{mg}$ per 100 gr fresh weight at 14 and 25 DAP, respectively. The highest levels of isoorientin are found in P1-rr;al, reaching 11 and $12 \mathrm{mg} / 100 \mathrm{gr}$ fresh weight, at 14 and 25 DAP, respectively, corresponding to a $\sim 8$ fold increase. In P1-rr, isoorientin increases 60 fold $(600 \mu \mathrm{g}$ per $100 \mathrm{gr}$ fresh weight) at $14 \mathrm{DAP}$ in contrast to a 110 fold increase $(240 \mu \mathrm{g}$ per $100 \mathrm{gr}$ fresh weight) at 25 DAP (Table 2, orange boxes). The orientin levels increase 6 and 8 fold at 14 and 25 DAP, respectively ( 56 and $13 \mu \mathrm{g} / 100 \mathrm{gr}$ fresh weight; Table 2, orange boxes). Overall, these results suggest that additional $O$-glycosylated flavones, products of alternative metabolic pathways likely not controlled by $P 1$, may be present in these lines (see Discussion).

\section{MAIZE WHOLE-FOOD EXTRACT PREPARATIONS INCREASED FLAVONE AGLYCONE CONTENT}

We previously showed that flavone aglycones have more anti-inflammatory activity than their glycosides counterparts (Hostetler et al., 2012). Hence, to evaluate whether food processing could increase the aglycone content in maize foods, we prepared whole maize extracts from kernels of the various genetic stocks using acid extraction [referred as AME (acid treated maize extracts)], or acid extraction combined with almond powder [referred as EME (enzyme treated maize extracts)], which we previously reported provides $\beta$-glucosidase activity, thereby increasing aglycone accumulation (Figure 5A) (Hostetler et al., 2012, 2013). Whole food extracts from P1-rr showed the highest apigenin content, reaching $\sim 77.4 \mu \mathrm{g} / 100 \mathrm{gr}$ fresh weight in AME and increasing $\sim 4$ fold in EME preparations to $\sim 241.1 \mu \mathrm{g} / 100 \mathrm{gr}$ fresh weight in EME (Figures 5B,C and Table 3). The almond powder contributes with negligible amounts of apigenin, as determined by HPLC analyses $(1.4 \mu \mathrm{g} / 100 \mathrm{gr}$ fresh weight) (Supplementary Figure 3). Both AME and EME foods from P1-rr;al contain significant less apigenin, ranging from 10 to $16 \mu \mathrm{g} / 100 \mathrm{gr}$ fresh weight, respectively (Figure 5, Table 3 ).

Surprisingly, P1-ww AME foods have $23.9 \mu \mathrm{g} / 100 \mathrm{gr}$ fresh weight of apigenin, increasing 5 fold to $\sim 103.9 \mu \mathrm{g} / 100 \mathrm{gr}$ fresh weight in EME preparations (Figures 5B,C). In contrast, sweet corn accumulates the lowest amount of apigenin in both AME and EME $(\sim 2.0$ and $8.2 \mu \mathrm{g} / 100 \mathrm{gr}$ fresh weight for each formulation respectively, Table 3 ). Collectively, these results suggest that preparations of foods using maize lines with increased flavone content significantly enhance the concentration of antiinflammatory forms of flavones lacking in commercial sweet corn.

\section{DISCUSSION}

In this study, we show that maize lines harboring the P1-rr allele in combination with recessive $a 1$ accumulate flavones at levels comparable to those present in celery, a vegetable often associated with the beneficial effects alleged to the Mediterranean's diet, and which is often presented as apigenin-rich (Hertog et al., 1992). Moreover, maize offers great advantages including the absence of other toxic compounds such as the linear furanocoumarins (e.g., psoralen, bergapten) that characterize celery, parsley and many of the other flavone-rich Apiaceaes and that can result in skin disorders, including cancer (Beier and Oertli, 1983; Diawara et al., 1995; Nigg et al., 1997). The ability to increase flavone content in crops of high agronomic relevance such as maize furnishes unique opportunities to improve nutrition. 


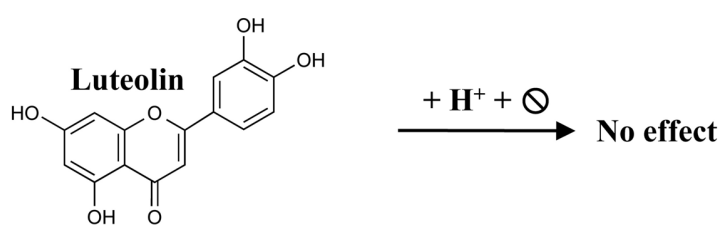

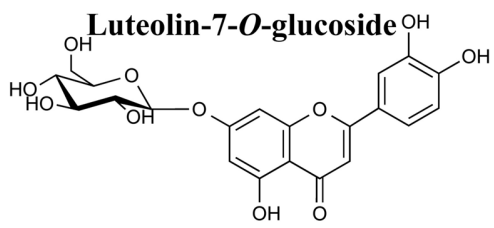<smiles>O=c1cc(-c2ccc(O)c(O)c2)oc2cc(O)cc(O)c12</smiles><smiles>COc1ccc(-c2cc(=O)c3c(O)cc(O)c(-c4ccc(O)c(O)c4)c3o2)c(O)c1</smiles>

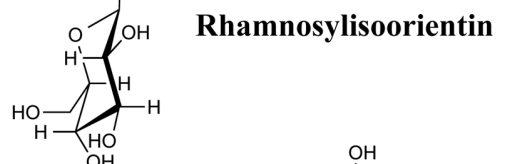

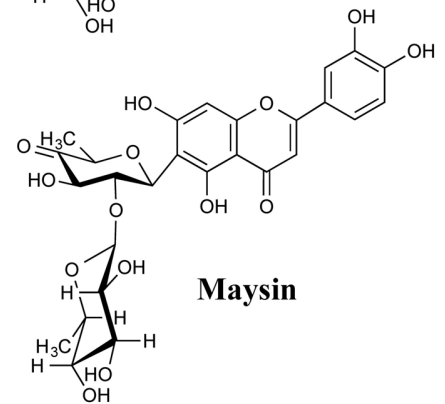

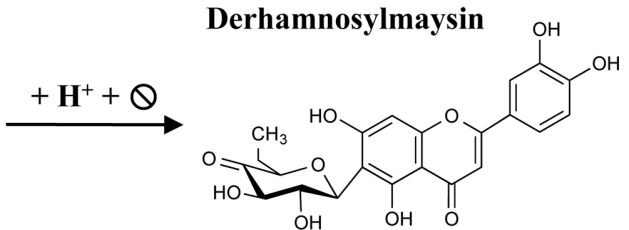

FIGURE 4 | Expected flavones resulting from acid hydrolysis. The scheme depicts the different flavones used in this study and their respective hydrolyzed derivatives expected for luteolin.

At 14 DAP in P1-rr and P1-rr;a1 maize kernels, about 95\% of all the flavones investigated here are present in the form of $C$-glucosides, $3 \%$ as $O$-glucosides and less than $0.1 \%$ as aglycones (Table 1). A number of biological activities have been attributed to $C$-glycosylflavones (Choi et al., 2013), including anti-diabetic, potential anti-Alzheimer's disease, and antiinflammatory activity, although aglycones are significantly more effective for the latter. Moreover, the economical value of $C$ glycosylated flavonoids is in the rise, as organic chemists are increasingly using these compounds as scaffolds for the generation of bioactive compounds because of their high biological activity potential (Talhi and Silva, 2012). However, it is not known to what extent $C$-glycosylflavones will be absorbed by the digestive system, or to what level gut microorganisms are able to process these compounds (Courts and Williamson, 2013). In contrast, $\mathrm{O}$-glycosylflavones are readily processed by the gut microbiota and are capable of releasing the respective aglycones (Courts and Williamson, 2013). In addition, during food processing, 

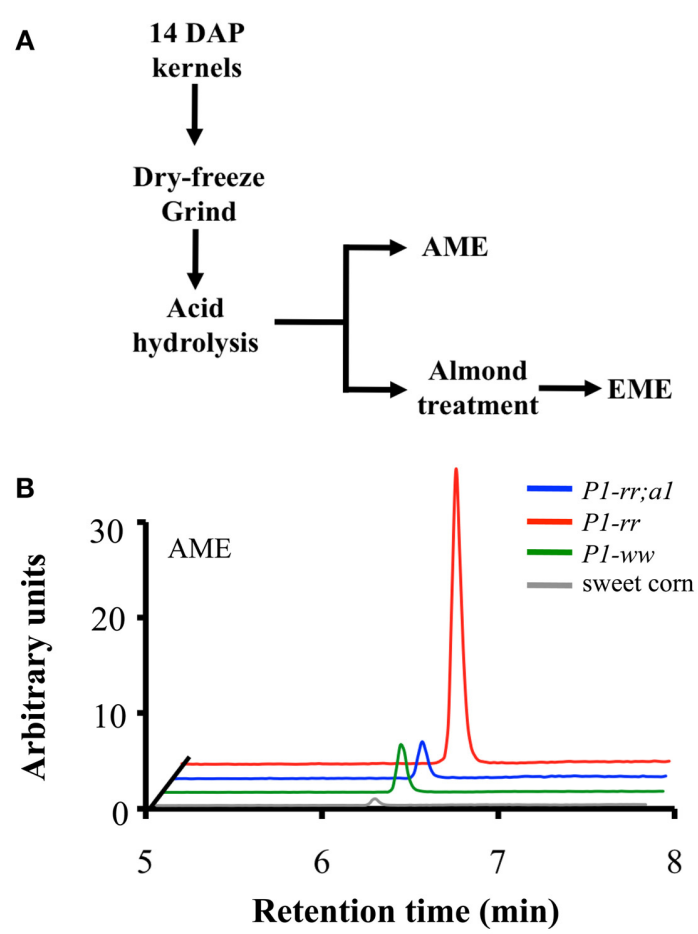

C

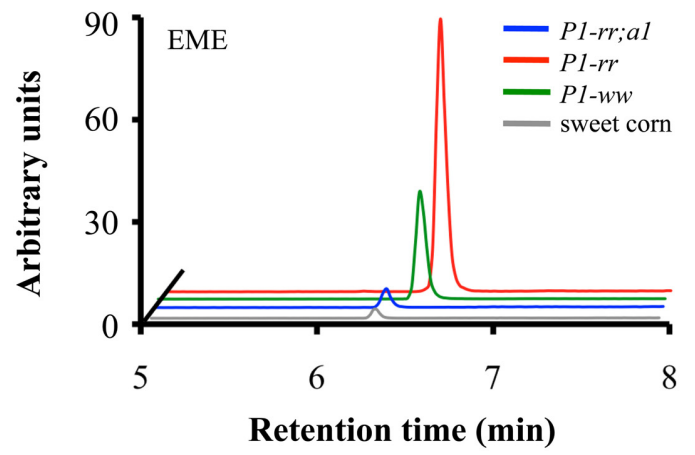

FIGURE 5 | Apigenin content in whole-food maize formulations. (A) Work flow of whole-maize kernel food extract preparation, AME: acid whole maize extract and EME: enzymatically treated whole maize extract. Chromatograms corresponding to apigenin content from P1-rr (red); P1-rr;a1 (blue); P1-ww (green) and sweet corn (gray) in AMEs (B) and EMEs (C). Arbitrary units correspond to peak intensity for the 268.9/117.0 transitions in counts per second.

particularly when using combinations of whole foods rich in glycosidases, an abundant release of the aglycones is observed (Figure 5). As previously noted, functional foods with increased aglycone content provide higher anti-inflammatory efficacy and increased absorption of flavonoids in vivo (Hostetler et al., 2012).

While the pericarp is part of the grain consumed as sweet corn, canned corn, and popcorn, it is eliminated by nixtamalization during the preparation of cornmeal for tortillas, tortilla chips and tamales. P1-rr kernels accumulate significantly larger flavone quantities than most lines commercially used, and the increase in free flavone and flavone $O$-glucoside accumulation in P1-rr;al offers attractive opportunities to further increase maize nutritional value. The absence of the DFR enzymatic activity in P1-rr;al lines diverts the naringenin intermediate toward flavone formation (Figure 1). Although a significant portion of the total flavones is used in the formation of $C$-glycosylflavones, a significant portion is utilized for the formation of flavone $O$-glucosides (Table 1), which can be hydrolyzed to the respective aglycones (Table 2). It is noteworthy, however, that hydrolysis did not completely eliminate the flavonoid $O$-glycosides (Table 2), something that was particularly puzzling in the case of $P 1-w w$ and the sweet corn line, in which the quantities of the flavones present fail to explain the levels observed after hydrolysis (Tables 1, 2). This likely indicates that there are a number of other flavone glycosides present in these maize lines that we failed to detect with our targeted MRM approach that could be identified through untargeted metabolomics analyses. A large number of flavones (more than 28 , including apigenin, apigenin-8- $C$-glucoside, vitexin and A7OG) were recently identified in a genome-wide association study of kernels from a large number of maize inbred lines using untargeted metabolomics (Wen et al., 2014). Perhaps one of these other flavones is responsible for the formation of small quantities of A7OG, L7OG, and isoorientin after hydrolysis. Additional studies will be required to determine if this is the case. There are multiple ways in which consumers could benefit from immature (14-20 DAP) high-flavone corn kernels, once the appropriate stocks are in the market, for example as popcorn, sweet corn, and canned corn. However, our studies also start providing some possible paths forward to introduce combinations of mutations that might significantly increase the accumulation of flavone in mature, dry kernels. Salmon silk $(\mathrm{Sm})$ genes (Anderson, 1921) are required for the formation of the major $C$ glycosylflavones present in maize floral organs (McMullen et al., 2004). Presumably, the combination of $a 1$ and $s m$ mutations in a P1-rr background will provide a further opportunity to increase the accumulation of hydrolyzable flavones in the maize kernel.

As previously shown in rice and wheat (Brazier-Hicks et al., 2009), and more recently in maize (Morohashi et al., 2012), the formation of $C$-glycosylflavones involves hydroxylation of a flavanone to a 2-hydroxyflavanone by $\mathrm{F} 2 \mathrm{H}$, which can then be C-glycosylated (Brazier-Hicks et al., 2009; Falcone Ferreyra et al., 2013). This poses the question of how apigenin or luteolin are formed, which presumably serve as the substrates for the respective $O$-glycosides. We speculate that there is a bona fide maize flavone synthase that can use naringenin (or eriodctyol) as substrates to form apigenin (or luteolin). Indeed, such an enzyme might not be significantly controlled by P1, since the levels of apigenin in P1-ww and P1-rr are comparable (Table 1). To determine to what extent the genes encoding the enzymes necessary for the formation of the flavanones are expressed in P1-ww, we analyzed recent RNA-Seq results obtained from pericarps with contrasting P1 alleles (Morohashi et al., 2012). We found that, albeit at low levels, two chalcone synthase genes (encoded by $C 2$ and WHP) and two chalcone isomerase genes (Supplementary Figure 4) are expressed in P1-ww pericarps at 14 and 25 DAP. These results suggest that a $P 1$-independent flavone accumulation pathway might be active in maize floral tissues.

In summary, our studies demonstrate that P1-rr maize kernels at 14 DAP potentially provide a good source of flavones 
Table 3 | Apigenin and luteolin contents in whole-food maize extracts.

\begin{tabular}{|c|c|c|c|c|c|c|c|c|}
\hline & \multicolumn{4}{|c|}{ AME } & \multicolumn{4}{|c|}{ EME } \\
\hline & P1-ww & P1-rr & P1-rr;a1 & Sweet Corn & P1-rr & P1-ww & P1-rr;a1 & Sweet corn \\
\hline Apigenin & 23.9 & 77.4 & 10.0 & 2.0 & 103.9 & 241.1 & 16.0 & 8.2 \\
\hline Luteolin & 15.6 & 184.4 & 279.3 & 5.0 & 18.9 & 300.6 & 274.3 & 7.6 \\
\hline
\end{tabular}

Apigenin and luteolin content was determined by LC-MS/MS using MRM in the negative mode following specific parental/daughter transitions (Figures $\mathbf{5 B}, \mathbf{C}$ ). Results are indicated as $\mu \mathrm{g}$ of apigenin/100 grams of fresh kernels.

that could complement flavone-deficient diets in the absence of other flavone-rich vegetables. The rich diversity offered by maize inbred lines and cultivars provide unique opportunities to select for maize genetic stocks that are simultaneously more resistant to emerging pathogens associated with global climate changes, and with increased levels of flavones with alleged beneficial effects and improved nutritional qualities.

\section{AUTHOR CONTRIBUTIONS}

Erich Grotewold, María I. Casas and Andrea I. Doseff carried out the experimental design. María I. Casas and Silvia Duarte performed the experiments. All authors analyzed data and contributed in the writing of the manuscript.

\section{ACKNOWLEDGMENT}

This work was supported in part by Agricultural and Food Research Initiative Competitive Grant 2010-65115-20408 from the US Department of Agriculture National Institute of Food and Agriculture to Erich Grotewold, by a grant from the National Science Foundation (IOS-1125620) to Andrea I. Doseff and Erich Grotewold, and the Plant Molecular Biology and Biotechnology Graduate Fellowship to María I. Casas.

\section{SUPPLEMENTARY MATERIAL}

The Supplementary Material for this article can be found online at: http://www.frontiersin.org/journal/10.3389/fpls.2014.00440/ abstract

\section{REFERENCES}

Anderson, E. G. (1921). The Inheritance of Salmon Color in Maize. Doctor of Philosophy Thesis, Cornell University, Ithaca, NY.

Anderson, E. G. (1924). Pericarp studies in maize II. The allelomorphism of a series of factors for pericarp color. Genetics 9, 442-453.

Anouar, E. H., Gierschner, J., Duroux, J., and Trouillas, P. (2012). UV/Visible spectra of natural polyphenols: a time-dependent density functional theory study. Food Chem. 131, 79-89. doi: 10.1016/j.foodchem.2011.08.034

Arango, D., Morohashi, K., Yilmaz, A., Kuramochi, K., Parihar, A., Brahimaj, B., et al. (2013). Molecular basis for the action of a dietary flavonoid revealed by the comprehensive identification of apigenin human targets. Proc. Natl. Acad. Sci. U.S.A. 110, E2153-E2162. doi: 10.1073/pnas. 1303726110

Bailey, D. M., and Bailey, R. M. (1938). The relation of the pericarp to tenderness in sweet corn. Proc. Am. Soc. Horti. 36, 555-559.

Beier, R. C., and Oertli, E. H. (1983). Psoralen and other linear furocoumarins as phytoalexins in celery. Phytochemistry 22, 2595-2597. doi: 10.1016/00319422(83)80173-3

Bligh, S. W. A., Ogegbo, O., and Wang, Z. (2013). "Flavonoids by HPLC," in Natural Products, eds K. G. Ramawat and J. M. Merillon (Berlin: Springer), 2107-2144.

Bonaccio, M., Iacoviello, L., De Gaetano, G., and Moli-Sani, I. (2011). The Mediterranean diet: the reasons for a success. Thromb. Res. 129, 401-404. doi: 10.1016/j.thromres.2011.10.018
Brazier-Hicks, M., Evans, K. M., Gershater, M. C., Puschmann, H., Steel, P. G., and Edwards, R. (2009). The C-glycosylation of flavonoids in cereals. J. Biol. Chem. 284, 17926-17934. doi: 10.1074/jbc.M109.009258

Byrne, P. F., McMullen, M. D., Wiseman, B. R., Snook, M. E., Musket, T. A., Theuri, J. M., et al. (1998). Maize silk maysin concentration and corn earworm antibiosis: QTLs and genetic mechanisms. Crop Sci. 38, 461-471. doi: 10.2135/cropsci1998.0011183X003800020032x

Byrne, P. F., McMullen, M. D., Snook, M. E., Musket, T. A., Theuri, J. M., Widstrom, N. W., et al. (1996). Quantitative trait loci and metabolic pathways: genetic control of the concentration of maysin, a corn earworm resistance factor, in maize silks. Proc. Natl. Acad. Sci. U.S.A. 93, 8820-8825. doi: 10.1073/pnas.93.17.8820

Casati, P., and Walbot, V. (2005). Differential accumulation of maysin and rhamnosylisoorientin in leaves of high altitude landraces of maize after UV-exposure. Plant Cell Environ. 28, 788-799. doi: 10.1111/j.1365-3040.2005.01329.x

Choi, J. S., Nurul Islam, M., Yousof Ali, M., Kim, E. J., Kim, Y. M., and Jung, H. A. (2013). Effects of C-glycosylation on anti-diabetic, anti-Alzheimer's disease and anti-inflammatory potential of apigenin. Food Chem. Toxicol. 64, 27-33. doi: 10.1016/j.fct.2013.11.020

Chopra, S., Athma, P., and Peterson, T. (1996). Alleles of the maize P gene with distinct tissue specificities encode Myb-homologous proteins with C-terminal replacements. Plant Cell 8, 1149-1158. doi: 10.1105/tpc.8.7.1149

Courts, F. L., and Williamson, G. (2013). The occurrence, fate and biological activities of C-glycosyl flavonoids in the human diet. Crit. Rev. Food Sci. Nutr. doi: 10.1080/10408398.2012.694497. [Epub ahead of print].

Diawara, M. M., Trumble, J. T., Quiros, C. F., and Hansen, R. (1995). Implications of distribution of linear furanocoumarins within celery. J. Agric. Food Chem. 43, 723-727. doi: 10.1021/jf00051a030

Duarte, S., Arango, D., Parihar, A., Hamel, P., Yasmeen, R., and Doseff, A. I. (2013). Apigenin protects endothelial cells from lipopolysaccharide (LPS)induced inflammation by decreasing caspase- 3 activation and modulating mitochondrial function. Int. J. Mol. Sci. 14, 17664-17679. doi: 10.3390/ijms140 917664

Falcone Ferreyra, M. L., Rius, S. P., and Casati, P. (2012). Flavonoids: biosynthesis, biological functions, and biotechnological applications. Front. Plant Sci. 3:222. doi: $10.3389 /$ fpls.2012.00222

Falcone Ferreyra, M. L., Rodriguez, E., Casas, M. I., Labadie, G., Grotewold, E., and Casati, P. (2013). Identification of a bifunctional maize Cand O-glucosyltransferase. J. Biol. Chem. 288, 31678-31688. doi: 10.1074/jbc.M113.510040

Ferder, L., Ferder, M. D., and Inserra, F. (2010). The role of high-fructose corn syrup in metabolic syndrome and hypertension. Curr. Hypertens. Rep. 12, 105-112. doi: 10.1007/s11906-010-0097-3

Gates, M. A., Vitonis, A. F., Tworoger, S. S., Rosner, B., Titus-Ernstoff, L., Hankinson, S. E., et al. (2009). Flavonoid intake and ovarian cancer risk in a population-based case-control study. Int. J. Cancer 124, 1918-1925. doi: 10.1002/ijc.24151

Gonzalez-Mejia, M. E., Voss, O. H., Murnan, E. J., and Doseff, A. I. (2010). Apigenin-induced apoptosis of leukemia cells is mediated by a bimodal and differentially regulated residue-specific phosphorylation of heat-shock protein-27. Cell Death Dis. 1, e64. doi: 10.1038/cddis.2010.41

Grotewold, E. (2006). The genetics and biochemistry of floral pigments. Annu. Rev. Plant Biol. 57, 761-780. doi: 10.1146/annurev.arplant.57.032905.105248

Grotewold, E., Athma, P., and Peterson, T. (1991a). A possible hot spot for Ac insertion in the maize P gene. Mol. Gen. Genet. 230, 329-331.

Grotewold, E., Athma, P., and Peterson, T. (1991b). Alternatively spliced products of the maize $\mathrm{P}$ gene encode proteins with homology to the DNA-binding 
domain of myb-like transcription factors. Proc. Natl. Acad. Sci. U.S.A. 88, 4587-4591.

Hertog, M. G. L., Hollman, P. C. H., and Venema, D. P. (1992). Optimization of a quantitative HPLC determination of potentially anticarcinogenic flavonoids in vegetables and fruits. J. Agric. Food Chem. 40, 1591-1598. doi: 10.1021/jf00021a023

Hostetler, G., Riedl, K., Cardenas, H., Diosa-Toro, M., Arango, D., Schwartz, S., et al. (2012). Flavone deglycosylation increases their anti-inflammatory activity and absorption. Mol. Nutr. Food Res. 56, 558-569. doi: 10.1002/mnfr.201100596

Hostetler, G. L., Riedl, K. M., and Schwartz, S. J. (2013). Effects of food formulation and thermal processing on flavones in celery and chamomile. Food Chem. 141, 1406-1411. doi: 10.1016/j.foodchem.2013.04.051

Huang, J., Pray, C., and Rozelle, S. (2002). Enhancing the crops to feed the poor. Nature 418, 678-684. doi: 10.1038/nature01015

Justesen, U., Knuthsen, P., and Leth, T. (1998). Quantitative analysis of flavonols, flavones, and flavanones in fruits, vegetables and beverages by high-performance liquid chromatography with photo-diode array and mass spectrometric detection. J. Chromatogr. A. 799, 101-110. doi: 10.1016/S00219673(97)01061-3

Kiesselbach, T. A. (1949). The Structure and Reproduction of Corn. Lincoln, NE: University of Nebraska.

Martin, C., Zhang, Y., Tonelli, C., and Petroni, K. (2013). Plants, diet, and health Annu. Rev. Plant Biol. 64, 19-46. doi: 10.1146/annurev-arplant-050312-120142

McMullen, M. D., Kross, H., Snook, M. E., Cortes-Cruz, M., Houchins, K. E., Musket, T. A., et al. (2004). Salmon silk genes contribute to the elucidation of the flavone pathway in maize (Zea mays L.). J. Hered. 95, 225-233. doi: 10.1093/jhered/esh042

Morohashi, K., Casas, M. I., Falcone Ferreyra, L., Mejia-Guerra, M. K., Pourcel, L., Yilmaz, A., et al. (2012). A genome-wide regulatory framework identifies maize pericarp color1 controlled genes. Plant Cell 24, 2745-2764. doi: 10.1105/tpc.112.098004

Morris, C. E., and Sands, D. C. (2006). The breeder's dilemma-yield or nutrition? Nat. Biotechnol. 24, 1078-1080. doi: 10.1038/nbt0906-1078

Nigg, H. N., Strandberg, J. O., Beier, R. C., Petersen, H. D., and Harrison, J. M. (1997). Furanocoumarins in Florida Celery varieties increased by Fungicide treatment. J. Agricult. Food Chem. 45, 1430-1436. doi: 10.1021/jf960537p

Patra, A. K., and Saxena, J. (2010). A new perspective on the use of plant secondary metabolites to inhibit methanogenesis in the rumen. Phytochemistry 71 1198-1222. doi: 10.1016/j.phytochem.2010.05.010

Pooma, W., Gersos, C., and Grotewold, E. (2002). Transposon insertions in the promoter of the Zea mays al gene differentially affect transcription by the Myb factors P and C1. Genetics 161, 793-801.

Sharma, M., Cortes-Cruz, M., Ahern, K. R., McMullen, M., Brutnell, T. P., and Chopra, S. (2011). Identification of the Prl gene product completes the anthocyanin biosynthesis pathway of maize. Genetics 188, 69-79. doi: 10.1534/ genetics.110.126136

Shukla, S., and Gupta, S. (2010). Apigenin: a promising molecule for cancer prevention. Pharm. Res. 27, 962-978. doi: 10.1007/s11095-010-0089-7

Singh, I., Sapna, L., and Pranjal, Y. (2014). Sweet corn and corn-based sweeteners. Sugar Tech. 16, 144-149. doi: 10.1007/s12355-014-0305-6

Snook, M. E., Widstrom, N. W., and Gueldner, R. C. (1989). Reversed-phase high-performance liquid chromatographic procedure fro the determination of maysin in corn silks. J. Chromatogr. A 477, 439-447. doi: 10.1016/S00219673(01)89654-0

Stafford, H. S. (1990). Flavonoid Metabolism. Boca Raton, FL: CRC Press.

Styles, D., and Ceska, O. (1989). Pericarp flavonoids in genetic strains of Zea mays. Maydica 34, 227-237.

Styles, E. D., and Ceska, O. (1977). The genetic control of flavonoid synthesis in maize. Can. J. Genet. Cytol. 19, 289-302.

Talhi, O., and Silva, A. M. S. (2012). Advances in C-glycosylflavonoid Research. Curr. Org. Chem. 16, 859-896. doi: 10.2174/138527212800194791

Tanumihardjo, S. A., Anderson, C., Kaufer-Horwitz, M., Bode, L., Emenaker, N. J., Haqq, A. M., et al. (2007). Poverty, obesity, and malnutrition: an international perspective recognizing the paradox. J. Am. Diet. Assoc. 107, 1966-1972. doi: 10.1016/j.jada.2007.08.007

Trichopoulou, A., and Vasilopoulou, E. (2000). Mediterranean diet and longevity. Br. J. Nutr. 84, S205-S209. doi: 10.1079/096582197388554

Vargo, M. A., Voss, O. H., Poustka, F., Cardounel, A. J., Grotewold, E., and Doseff, A. I. (2006). Apigenin-induced-apoptosis is mediated by the activation of PKCdelta and caspases in leukemia cells. Biochem. Pharmacol. 72, 681-692. doi: 10.1016/j.bcp.2006.06.010

Vasta, V., and Luciano, G. (2011). The effects of dietary consumption of plant secondary compounds on small ruminants' products quality. Small Ruminant Res. 101, 150-159. doi: 10.1016/j.smallrumres.2011.09.035

Wahrburg, U., Kratz, M., and Cullen, P. (2002). Mediterranean diet, olive oil and health. Eur. J. Lipid Sci. Tech. 104, 698-705. doi: 10.1002/1438-9312(200210) 104:9/10<698::AID-EJLT698>3.0.CO;2-A

Wen, W., Li, D., Gao, Y., Li, W., Liu, J., Liu, H., et al. (2014). Metabolome-based genome-wide association study of kernel leads to novel biochemical insights. Nat. Commun. 5:3438. doi: 10.1038/ncomms 4438

Winkel-Shirley, B. (2001). Flavonoid biosynthesis. A colorful model for genetics, biochemistry, cell biology, and biotechnology. Plant Physiol. 126, 485-493. doi: 10.1104/pp.126.2.485

Yan, J., Yu, L., Shuang, X., Gu, W., and Zhu, W. (2014). Apigenin accumulation and expression analysis of apigenin biosynthesis relative genes in celery. Sci. Horti. 165, 218-224. doi: 10.1016/j.scienta.2013.11.018

Conflict of Interest Statement: The authors declare that the research was conducted in the absence of any commercial or financial relationships that could be construed as a potential conflict of interest.

Received: 28 June 2014; accepted: 16 August 2014; published online: 08 September 2014

Citation: Casas MI, Duarte S, Doseff AI and Grotewold E (2014) Flavone-rich maize: an opportunity to improve the nutritional value of an important commodity crop. Front. Plant Sci. 5:440. doi: 10.3389/fpls.2014.00440

This article was submitted to Plant Metabolism and Chemodiversity, a section of the journal Frontiers in Plant Science.

Copyright (๑) 2014 Casas, Duarte, Doseff and Grotewold. This is an open-access article distributed under the terms of the Creative Commons Attribution License (CC BY). The use, distribution or reproduction in other forums is permitted, provided the original author(s) or licensor are credited and that the original publication in this journal is cited, in accordance with accepted academic practice. No use, distribution or reproduction is permitted which does not comply with these terms. 